\title{
Association between Weight Changes after Smoking Cessation and Cardiovascular Disease among the Korean Population
}

\author{
Eun Ha Kim, Hyejin Lee*, Dong Wook Shin, Jae Moon Yun, Jung-Hyun Shin, Yoo Kyoung Lim, Hye Yeon Koo, \\ Miso Jang \\ Department of Family Medicine, Seoul National University Hospital, Seoul, Korea
}

\begin{abstract}
Background: Cigarette smoking is a risk factor for cardiovascular disease (CVD) and has both beneficial and harmful effects in CVD. We hypothesized that weight gain following smoking cessation does not attenuate the CVD mortality of smoking cessation in the general Korean population.

Methods: Study subjects comprised 2.2\% randomly selected patients from the Korean National Health Insurance Corporation, between 2002 and 2013. We identified 61,055 subjects who were classified as current smokers in 2003-2004. After excluding 21,956 subjects for missing data, we studied 30,004 subjects. We divided the 9,095 exsmokers into two groups: those who gained over $2 \mathrm{~kg}(2,714)$, and those who did not gain over $2 \mathrm{~kg}(6,381$, including weight loss), after smoking cessation. Cox proportional hazards regression models were used to estimate the association between weight gain following smoking cessation and CVD mortality.

Results: In the primary analysis, the hazard ratios of all-cause deaths and CVD deaths were assessed in the three groups. The CVD risk factors and Charlson comorbidity index adjusted hazard ratios (aHRs) for CVD deaths were 0.80 (95\% confidence interval [CI], 0.37 to 1.75 ) for ex-smokers with weight gain and 0.80 ( $95 \%$ CI, 0.50 to 1.27 ) for ex-smokers with no weight gain, compared to one for sustained smokers. The associations were stronger for events other than mortality. The aHRs for CVD events were 0.69 ( $95 \%$ CI, 0.54 to 0.88 ) and 0.81 (95\% CI, 0.70 to 0.94 ) for the ex-smokers with and without weight gain, respectively.

Conclusion: Although smoking cessation leads to weight gain, it does not increase the risk of CVD death.
\end{abstract}

Keywords: Cardiovascular Diseases; Smoking Cessation; Weight Gain; Risk Factors 


\section{INTRODUCTION}

Cigarette smoking is not only associated with cancer, but is also one of the best-known modifiable risk factors for cardiovascular disease (CVD). ${ }^{1,2)}$ Cigarette smoking affects endothelium-dependent vasodilatation; therefore, it mediates inflammation, dyslipidemia, platelet dysfunction, and coagulation problems. Additionally, smoking increases the incidences of hypertension and diabetes. ${ }^{3,4}$

Smoking cessation reduces CVD risk; ${ }^{5)}$ however, weight gain following smoking cessation is one of the reasons people either do not try to quit or relapse following smoking cessation. ${ }^{6,7)}$ In addition, weight gain itself is a risk factor for $\mathrm{CVD}^{8}{ }^{8)}$ Vascular mortality increases $40 \%$ for every five-unit increase in body mass index (BMI) above 25. ${ }^{9)}$ The mean weight gain after smoking cessation varies between 3 and $6 \mathrm{~kg}$ in North America and Asia. This weight gain generally occurs within six months after smoking cessation, and it persists over time. ${ }^{6,10-14)}$ Therefore, there is some concern that weight gain after smoking cessation might attenuate the CVD-related benefits of quitting smoking.

Few previous studies have examined the association between weight gain after smoking cessation and CVD. Some of these have shown that smoking cessation is associated with a lower risk of $\mathrm{CVD}^{10,11,15)}$ despite weight gain; however, these studies have some limitations. First, most of them were conducted in the West, ${ }^{10,15)}$ and there are very few studies regarding this issue in Asia. Second, all these studies assessed the effects of weight gain following smoking cessation on CVD indirectly. For example, one study calculated changes in estimated CVD incidence risk following weight gain using BMI, systolic blood pressure (SBP), and total cholesterol, instead of the actual incidence count itself. ${ }^{11)}$ Another study analyzed the associations between weight gain after smoking cessation and CVD by comparing hazard ratio differences between weight changes. This included adjusted hazard ratios (aHRs) and only adjusted for CVD risk factors. ${ }^{10)}$

Therefore, the aim of this study was to assess the associations between weight gain following smoking cessation and CVD mortality directly. We hypothesized that smoking cessation would decrease CVD mortality risk compared to continued smoking, despite the associated weight gain.

\section{METHODS}

\section{Screening Program in Korea}

In South Korea, there is universal healthcare coverage, the Korean National Health Insurance (KNHI) Corporation, which is compulsory for all citizens in South Korea to participate. Currently, the National Health Insurance Service (NHIS) maintains and manages all databases of Korea's health service utilization. ${ }^{16)}$

The NHIS announced a National Sample Cohort (NSC) database (The NHIS-NSC 2002-2010) in July 2014, which included 1,025,340 Koreans as an initial 2002 cohort. These subjects underwent follow-up for nine years, until 2010. The cohort data randomly sampled about $2.2 \%$ of Korea's total population in $2002(46,605,433)$ to represent an individual's total annual medical expenses within each of 1,476 strata, as defined by anthropological characteristics: age, sex, eligibility status (employed or self-employed), and income level (20 quartiles for each eligibility status and medical aid beneficiary) combinations. This cohort study was constructed semi-dynamically wherein participant follow-up lasted until death, emigration, or the end of the study period, while samples of newborn infants were added annually. The database contains participant eligibility and demographic information regarding health insurance and medical aid beneficiaries, medical bill details, medical treatment and disease histories, and prescriptions. Such data were constructed after converting insurance claim information to the first day of medical treatment.

In the cohort, the subject's mortality information along with cause of death was included. The latter was classified according to the 10th revision of the International Classification of Diseases (ICD-10) codes, which was obtained from the Korean National Statistical Office. Furthermore, the laboratory and survey questionnaire data of cardiovascular and life-transition health screening for all cohort members were merged.

\section{Data Collection}

The KNHI Corporation recommends a biennial health-screening program for all KNHI members. KNHI collects medical claims and demographic data, including age, sex, monthly insurance premium, disability status, disease codes, and costs incurred. Medical history was based on the diagnoses in $\mathrm{KNHI}$ medical service claims data during 2002, coded using the ICD-10. ${ }^{17)}$ Comorbidities were calculated using the Charlson comorbidity index, a weighted measure of comorbidity. The index is composed of 19 conditions, including previous CVD, heart failure, and cancers. ${ }^{18,19)}$

We obtained screening data of participants from the KNHI screening database. Health questionnaires included information on smoking (non-smoker, ex-smoker, and current smoker), alcohol consumption (drinking habit, times/wk) and medical history (hypertension and diabetes). Physical exams included measurements of weight, height, and SBP. BMI was calculated as weight in kilograms divided by height in squared meters. Laboratory tests included total cholesterol and fasting blood glucose.

\section{Participants}

We used data from all KNHI members who underwent a screening examination between January 2002 and December 2013 (2002-2013 cohort observation, $\mathrm{N}=1,125,691)$. From these, we identified 61,055 subjects who were classified as current smokers in 2003-2004. As the screening was provided biennially, we used two-year windows (e.g., 2003-2004 or 2005-2006) to define screening program participations. All participants were classified as current smokers, ex-smokers, or non-smokers based on self-reported questionnaires completed at each examination.

We excluded participants with CVD diagnosed before 2007. In our study, CVD was defined as coronary heart disease (CHD) and stroke. 
We also excluded participants who died or were censored before 2007, and for whom there was no information about age, sex, weight, height, SBP, total cholesterol, fasting blood glucose, drinking habits, or smoking status for 2005-2006. Among the 61,055 participants, 21,956 were excluded, and data were analyzed for the remaining 39,099.

The 39,099 subjects were smokers at baseline, 2003-2004. Among them, 30,004 were still smoking in 2005-2006, and 9,095 had stopped smoking. We divided the ex-smokers into two groups: those who gained over $2 \mathrm{~kg}$ after smoking cessation, and those who did not gain over $2 \mathrm{~kg}$ (some of them even lost weight). The latter group was named the no weight gain group (Figure 1). The cutoff point for weight gain of over $2 \mathrm{~kg}$ was determined based on previous studies, since $2 \mathrm{~kg}$ is the typical weight gain following smoking cessation in Asia. ${ }^{11,20)}$ However, weight gain of over $5 \mathrm{~kg}$ might increase the risk of CVD mortality.

\section{Study Outcomes}

The primary outcome for this study was CVD mortality. We defined CVD as a composite of CHD, including myocardial infarction (ICD-10 codes I21-I22) and stroke (ischemic and hemorrhagic stroke, I60-I64). The secondary outcomes were all-cause mortality and CVD events. Vital status was assured routinely by matching KNHI data with the National Death Registry. CVD deaths were defined as deaths with underlying ICD-10 codes I00-I99, as registered in the National Death Registry.

\section{Statistical Analysis}

To assess weight changes following smoking cessation, we used the data of 2003-2004 smokers who had stopped smoking by 2005-2006. We divided the 2005-2006 ex-smokers into two groups, as described above. Finally, for the analysis, we divided the participants into three groups: sustained smokers, ex-smokers who gained over $2 \mathrm{~kg}$, and exsmokers who did not gain weight. Smokers were chosen as the reference group to assess the association between quitting smoking and CVD. In the analyses, the association between smoking cessation after weight gain ( $>2 \mathrm{~kg}$ ) and mortality (CVD and all-cause) was estimated using Cox proportional-hazards regression models. Follow-up extended until whichever of the following came first: CVD endpoint development, death, or December 31, 2013. Additionally, we reviewed the risk of CVD mortality for over $5 \mathrm{~kg}$ weight gain.

The calculation of aHRs was done after adjusting CVD risk factors (age, sex, BMI, SBP, total cholesterol, fasting blood glucose, drinking habits, medical history of hypertension, and diabetes) and Charlson comorbidity index.

We performed a chi-square test or analysis of covariance to compare the basic characteristics between smokers and ex-smokers (who gained weight over $2 \mathrm{~kg}$ and who did not gain weight). Statistical analyses were performed in Stata SE ver. 14.0 (Stata Corp., College Station, TX, USA). Statistical significance was defined as two-tailed P-values of $<0.05$.

Total cohort for observation $\mathrm{N}=1,125,691$ (censored 2002-2013)

Smokers in 2003-2004 $(n=61,055)$

Missing data in 2005-2006

Missing data of fasting blood glucose, total cholesterol, systolic blood pressure, drinking habits, smoking status, height, $n=20,332$ excluded

(No missing data of Charlson comorbidity index, sex, age, weight, and medical history of hypertension and diabetes.)

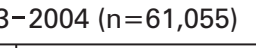




\section{RESULTS}

Of the 39,099 study participants, 30,004 were still smoking, and 9,095 had quit smoking by 2005-2006. Among the 9,095 ex-smokers, 2,714 (29.8\%) gained weight ( $>2 \mathrm{~kg}$ ) while the remaining 6,381 (70.2\%) did not. The participant's basic characteristics are described in Table 1. Proportionally, there were few female subjects. Because of the large sex distribution difference, we confirmed whether there was a difference in the results after including and excluding the data obtained from women. In our analysis, we assessed the association between weight gain following smoking cessation and CVD mortality only for men. However, there was little difference between them, and the result was unchanged, so we included the data obtained from women in our final analysis.

Ex-smokers who did not gain weight tended to have a more frequent medical history of hypertension and diabetes (hypertension, $2.79 \%$; diabetes, $1.38 \%$ ), compared to ex-smokers who gained weight (hypertension, $1.62 \%$; diabetes, $0.77 \%$ ). Sustained smokers tended to drink

Table 1. Participants characteristics in 2005-2006

\begin{tabular}{|c|c|c|c|c|}
\hline \multirow{2}{*}{ Characteristic } & \multirow{2}{*}{ Total no. } & \multirow{2}{*}{ Sustained smoker } & \multicolumn{2}{|c|}{ Ex-smoker } \\
\hline & & & Weight gain (>2 kg) & No weight gain \\
\hline No. of participants & 39,099 & 30,004 & 2,714 & 6,381 \\
\hline \multicolumn{5}{|l|}{ Age group (y) } \\
\hline$<40$ & $18,825(48.15)$ & $14,946(49.81)$ & $1,400(51.58)$ & 2,479 (38.85) \\
\hline $40-49$ & 11,194 (28.63) & $8,559(28.53)$ & $664(24.47)$ & $1,971(30.89)$ \\
\hline $50-59$ & $5,623(14.38)$ & 4,079 (13.59) & $407(15.00)$ & $1,137(17.82)$ \\
\hline $60-69$ & $2,579(6.60)$ & $1,843(6.14)$ & $166(6.12)$ & $570(8.93)$ \\
\hline$\geq 70$ & $878(2.25)$ & 577 (1.92) & $77(2.84)$ & $224(3.51)$ \\
\hline Women & $1,143(2.92)$ & $634(2.11)$ & $116(4.27)$ & $393(6.16)$ \\
\hline \multicolumn{5}{|l|}{ Drinking habits (time/wk) } \\
\hline$<1$ & 19,507 (49.89) & $13,410(44.69)$ & $1,743(64.22)$ & $4,354(68.23)$ \\
\hline $1-2$ & $13,390(34.25)$ & $11,259(37.52)$ & 685 (25.24) & $1,446(22.66)$ \\
\hline $3-4$ & 4,605 (11.78) & 3,942 (13.14) & $225(8.29)$ & $438(6.836$ \\
\hline Almost every day & $1,597(4.08)$ & $1,393(4.64)$ & $61(2.25)$ & $143(2.24)$ \\
\hline \multicolumn{5}{|l|}{ Past medical history } \\
\hline Hypertension & 935 (2.39) & $713(2.38)$ & 44 (1.62) & $178(2.79)$ \\
\hline Diabetes & $504(1.29)$ & $395(1.32)$ & $21(0.77)$ & 88 (1.38) \\
\hline Body mass index* & & $23.85 \pm 3.07$ & $24.54 \pm 2.97$ & $23.83 \pm 2.96$ \\
\hline Systolic blood pressure (mm Hg) & & $124.17 \pm 14.50$ & $125.06 \pm 15.12$ & $124.67 \pm 15.16$ \\
\hline Total cholesterol (mg/dL) & & $193.76 \pm 36.14$ & $197.59 \pm 36.28$ & $194.64 \pm 36.06$ \\
\hline Fasting blood glucose (mg/dL) & & $95.70 \pm 24.59$ & $94.15 \pm 19.42$ & $96.69 \pm 26.66$ \\
\hline Charlson comorbidity index & & $0.34 \pm 0.68$ & $0.37 \pm 0.70$ & $0.46 \pm 0.88$ \\
\hline
\end{tabular}

Values are presented as number (\%) or mean \pm standard deviation.

${ }^{*}$ Calculated as weight in kilograms divided by height in meters squared.

Table 2. Association between weight change following smoking cessation and CVD mortality as well as all-cause mortality

\begin{tabular}{|c|c|c|c|c|}
\hline \multirow{2}{*}{ Variable } & \multirow{2}{*}{ Total $(\mathrm{N}=39,099)$} & \multirow{2}{*}{ Sustained smoker } & \multicolumn{2}{|c|}{ Ex-smoker } \\
\hline & & & Weight gain (>2 kg) & No weight gain \\
\hline No. of all cause death & 1,068 & 791 & 65 & 212 \\
\hline Unadjusted HR & & Reference & $0.91(0.70-1.17)$ & $1.26(1.09-1.47)$ \\
\hline Adjusted $\mathrm{HR}^{*}$ & & Reference & $0.92(0.71-1.97)$ & $0.88(0.76-1.03)$ \\
\hline No. of $\mathrm{CVD}^{\dagger}$ death & 121 & 91 & 7 & 23 \\
\hline Unadjusted HR & & Reference & 0.85 (0.39-1.83) & $1.19(0.75-1.88)$ \\
\hline Adjusted HR & & Reference & $0.80(0.37-1.75)$ & $0.80(0.50-1.27)$ \\
\hline No. of CHD death & 50 & 41 & 2 & 7 \\
\hline Unadjusted HR & & Reference & $0.54(0.13-2.23)$ & $0.80(0.36-1.79)$ \\
\hline Adjusted HR & & Reference & $0.49(0.12-2.05)$ & $0.61(0.27-1.38)$ \\
\hline No. of stroke death & 71 & 50 & 5 & 16 \\
\hline Unadjusted HR & & Reference & $1.10(0.44-2.77)$ & $1.51(0.86-2.65)$ \\
\hline Adjusted HR & & Reference & $1.11(0.44-2.83)$ & $0.93(0.52-1.66)$ \\
\hline
\end{tabular}

Values are presented as number or HR (95\% confidence interval).

CVD, cardiovascular disease; HR, hazard ratio; CHD, coronary heart disease.

*Adjusted for CVD risk factors: age, sex, body mass index, systolic blood pressure, total cholesterol, fasting blood glucose, drinking habits, past medical history of hypertension and diabetes, and Charlson comorbidity index. ${ }^{\dagger} \mathrm{CVD}$ comprises CHD and stroke. 
more (Table 1).

Ex-smokers tended to have lower CVD mortality risk. Furthermore, despite weight gain, ex-smokers had less risk of death from any cause compared to sustained smokers (Table 2), although the differences were not significant. After adjusting for covariates, ex-smokers still had a lower CVD mortality risk regardless of weight gain, although the results were statistically insignificant. The CVD risk factors and Charlson comorbidity index aHRs for all-cause death were 0.92 (95\% confidence interval [CI], 0.71 to 1.97) and 0.88 (95\% CI, 0.76 to 1.03) for ex-smokers who gained weight and those who did not, respectively (Table 2). Only the CVD risk factors and Charlson comorbidity index aHRs for stroke mortality were higher among ex-smokers who gained weight, but the 95\% CI was too wide to confirm due to the small numbers of casualties.

During follow-up, 1,343 CVD events occurred. We analyzed HRs for the CVD events to evaluate the associations between weight gain following smoking cessation and CVD risk. The associations were stronger with events other than mortality. The CVD risk factors and Charlson comorbidity index aHRs for the CVD events were 0.69 (95\% CI, 0.54 to 0.88 ) and 0.81 (95\% CI, 0.70 to 0.94 ) for the ex-smokers who gained weight and those who did not, respectively (Table 3 ).

As previously mentioned, weight gain of over $5 \mathrm{~kg}$ might increase CVD mortality and all-cause mortality risk compared to sustained smokers. The CVD risk factors and Charlson comorbidity index aHRs for all causes death were 1.21 (95\% CI, 0.77 to 1.90) and 0.85 (95\% CI, 0.73 to 0.99 ) for ex-smokers who gained weight and those who did not, respectively, although the differences were not statistically significant (Appendix 1). As for CVD events, the results were not markedly different from the $2 \mathrm{~kg}$ cutoff for weight gain (Appendix 2).

\section{DISCUSSION}

In our study, we used the NSC (2002-2013) database to assess the associations between weight gain after smoking cessation and CVD mortality. Therefore, our study represents the general Korean population. We found that ex-smokers tended to have lower CVD mortality risk. Furthermore, there was less death risk from all causes despite weight gain following smoking cessation. In addition, ex-smokers had significantly lower risks of CVD events compared to sustained smokers, despite weight gain.

One community-based cohort study of the associations between weight gain following smoking cessation and CVD conducted in the United States found that there was an association between smoking cessation and lower CVD risk that was not modified by weight gain. The aHR for CVD risk factors and weight change was 0.61 (95\% CI, 0.21 to 1.78 ) for $\leq 4$ years quitters, 0.29 ( $95 \% \mathrm{CI}, 0.16$ to 0.52 ) for $>4$ years quitters (for smokers, this value was 1 ). ${ }^{10)}$ A similar study was conducted in Japan in 2010. Among the male working population, weight gain after smoking cessation was approximately $2 \mathrm{~kg}$, and the estimated CHD risk four years later was 0.76 (95\% CI, 0.68 to 0.85 ), compared to sustained smokers. ${ }^{11)}$

However, these studies were not without limitations. They did not assess the effects of weight gain following smoking cessation in association with CVD events or mortality itself. Instead, they assessed the effect through weight gain aHR or estimated CVD risk. In addition, the study conducted in Japan included only Japanese male workers; therefore, they were unable to apply the results to the general population. The offspring cohort of the Framingham Heart Study had as long as a follow-up period as presented here; however, these participants were mainly Caucasians.

Table 3. Association between weight change following smoking cessation and CVD events

\begin{tabular}{|c|c|c|c|c|}
\hline \multirow{2}{*}{ Variable } & \multirow{2}{*}{ Total $(\mathrm{N}=39,099)$} & \multirow{2}{*}{ Sustained smoker } & \multicolumn{2}{|c|}{ Ex-smoker } \\
\hline & & & Weight gain (>2 kg) & No weight gain \\
\hline No. of CVD* events & 1,343 & 1,019 & 74 & 250 \\
\hline Unadjusted HR & & Reference & $0.80(0.63-1.01)$ & $1.16(1.01-1.33)$ \\
\hline Adjusted $\mathrm{HR}^{\dagger}$ & & Reference & $0.69(0.54-0.88)$ & $0.81(0.70-0.94)$ \\
\hline No. of CHD events & 831 & 626 & 44 & 161 \\
\hline Unadjusted HR & & Reference & $0.78(0.57-1.05)$ & $1.12(1.02-1.45)$ \\
\hline Adjusted HR & & Reference & $0.68(0.50-0.93)$ & $0.91(0.76-1.08)$ \\
\hline No. of stroke $e^{\ddagger}$ events & 557 & 428 & 33 & 96 \\
\hline Unadjusted HR & & Reference & $0.85(0.60-1.21)$ & $1.06(0.85-1.32)$ \\
\hline Adjusted HR & & Reference & $0.76(0.53-1.08)$ & $0.73(0.58-0.92)$ \\
\hline No. of ischemic stroke events & 426 & 328 & 24 & 74 \\
\hline Unadjusted HR & & Reference & $0.81(0.53-1.22)$ & $1.07(0.83-1.37)$ \\
\hline Adjusted HR & & Reference & $0.71(0.47-1.08)$ & $0.71(0.55-0.92)$ \\
\hline No. of hemorrhagic stroke events & 142 & 109 & 9 & 24 \\
\hline Unadjusted HR & & Reference & $0.91(0.46-1.80)$ & $1.04(0.67-1.62)$ \\
\hline Adjusted HR & & Reference & $0.83(0.42-1.66)$ & $0.79(0.50-1.24)$ \\
\hline
\end{tabular}

Values are presented as number or HR (95\% confidence interval).

CVD, cardiovascular disease; HR, hazard ratio; CHD, coronary heart disease.

${ }^{*}$ CVD comprises CHD and stroke. ${ }^{\dagger}$ Adjusted for CVD risk factors: age, sex, body mass index, systolic blood pressure, total cholesterol, fasting blood glucose, drinking habits, past medical history of hypertension and diabetes, and Charlson comorbidity index. ${ }^{\ddagger}$ Stroke comprises ischemic stroke and hemorrhagic stroke. 
One Korean study analyzed the associations between weight gain after smoking cessation and CVD risk factors. ${ }^{20)}$ In this study, the quitters were more likely to have higher SBP, as well as a higher total and low-density lipoprotein cholesterol than sustained smokers, which could be secondary to weight gain following smoking cessation. The quitters who gained more than $1.3 \mathrm{~kg}$ were more likely to have increased blood pressure, as well as higher total cholesterol, triglycerides, non-high-density lipoprotein cholesterol, and fasting glucose levels. This study's limitation was that it focused on surrogate variables, not on CVD itself, and the mean follow-up duration was relatively short (1-3 years).

Our study has several limitations. First, most of the results were not significant due to the small number of events. In this study, we followed up with mortality and other events from 2007 to 2013, which is a relatively short follow-up period for survival analysis. Therefore, future studies with longer follow-up periods may provide significant results. Second, our study could have selection bias. People who participate in biennial health screening programs tend to be more concerned about their health. Thus, participants who quit smoking and participate in biennial health screening programs may have lower CVD mortality and fewer events. Third, we could not include all potential confounding factors in our analysis, such as physical activity, duration and amount of smoking, restarting smoking and dietary habits. Since these factors are usually self-reported subjective measurements, bias may be present. Furthermore, resuming smoking may have affected the results of this study, although we did not adjust for resuming smoking. Since ex-smokers commonly resume smoking, yearly smoking status is a floating variable. If we had adjusted for yearly smoking status, there would be many more excluded subjects. For this reason, we did not adjust for the resumption of smoking. Furthermore, the reduction in CVD mortality and events may have resulted from changes in lifestyle factors other than smoking cessation, as it appears participants who stop smoking are less likely to drink alcohol than are sustained continuers. Fourth, we used $2 \mathrm{~kg}$ as the cutoff point for weight gain based on previous studies, because $2 \mathrm{~kg}$ is the typical weight gain following smoking cessation in Asia. However, clinically significant weight gain might increase the risk of CVD mortality.

In conclusion, this study suggests that, although smoking cessation may result in weight gain, this risk does not outweigh the benefits of smoking cessation in terms of CVD mortality risk reductions. Healthcare providers can use this information to improve smoking cessation programs, in which people seek advice. It should be stressed that quitting smoking is a healthier choice, even if weight is gained as a result of the cessation.

\section{CONFLICT OF INTEREST}

No potential conflict of interest relevant to this article was reported.

\section{REFERENCES}

1. Kannel WB. Update on the role of cigarette smoking in coronary artery disease. Am Heart J 1981;101:319-28.

2. Jee SH, Suh I, Kim IS, Appel LJ. Smoking and atherosclerotic cardiovascular disease in men with low levels of serum cholesterol: the Korea Medical Insurance Corporation Study. JAMA 1999;282:2149-55.

3. Jee SH, Foong AW, Hur NW, Samet JM. Smoking and risk for diabetes incidence and mortality in Korean men and women. Diabetes Care 2010;33:2567-72.

4. Bowman TS, Gaziano JM, Buring JE, Sesso HD. A prospective study of cigarette smoking and risk of incident hypertension in women. J Am Coll Cardiol 2007;50:2085-92.

5. Jha P. The 21st century benefits of smoking cessation in Europe. Eur J Epidemiol 2013;28:617-9.

6. Filozof C, Fernandez Pinilla MC, Fernandez-Cruz A. Smoking cessation and weight gain. Obes Rev 2004;5:95-103.

7. Pistelli F, Aquilini F, Carrozzi L. Weight gain after smoking cessation. Monaldi Arch Chest Dis 2009;71:81-7.

8. Jousilahti P, Tuomilehto J, Vartiainen E, Pekkanen J, Puska P. Body weight, cardiovascular risk factors, and coronary mortality: 15-year follow-up of middle-aged men and women in eastern Finland. Circulation 1996;93:1372-9.

9. Prospective Studies Collaboration, Whitlock G, Lewington S, Sherliker P, Clarke R, Emberson J, et al. Body-mass index and cause-specific mortality in 900000 adults: collaborative analyses of 57 prospective studies. Lancet 2009;373:1083-96.

10. Clair C, Rigotti NA, Porneala B, Fox CS, D’Agostino RB, Pencina MJ, et al. Association of smoking cessation and weight change with cardiovascular disease among adults with and without diabetes. JAMA 2013;309:1014-21.

11. Tamura U, Tanaka T, Okamura T, Kadowaki T, Yamato H, Tanaka H, et al. Changes in weight, cardiovascular risk factors and estimated risk of coronary heart disease following smoking cessation in Japanese male workers: HIPOP-OHP study. J Atheroscler Thromb 2010;17:12-20.

12. Froom P, Melamed S, Benbassat J. Smoking cessation and weight gain. J Fam Pract 1998;46:460-4.

13. Williamson DF, Madans J, Anda RF, Kleinman JC, Giovino GA, Byers T. Smoking cessation and severity of weight gain in a national cohort. $\mathrm{N}$ Engl J Med 1991;324:739-45.

14. Pisinger C, Jorgensen T. Waist circumference and weight following smoking cessation in a general population: the Inter99 study. Prev Med 2007;44:290-5.

15. Siahpush M, Singh GK, Tibbits M, Pinard CA, Shaikh RA, Yaroch A. It is better to be a fat ex-smoker than a thin smoker: findings from the 1997-2004 National Health Interview Survey-National Death Index linkage study. Tob Control 2014;23:395-402.

16. Lee H, Cho J, Shin DW, Lee SP, Hwang SS, Oh J, et al. Association of cardiovascular health screening with mortality, clinical outcomes, and health care cost: a nationwide cohort study. Prev Med 2015;70:19-25.

17. Wilchesky M, Tamblyn RM, Huang A. Validation of diagnostic codes within medical services claims. J Clin Epidemiol 2004;57:131-41.

18. Klabunde CN, Legler JM, Warren JL, Baldwin LM, Schrag D. A refined comorbidity measurement algorithm for claims-based studies of breast, prostate, colorectal, and lung cancer patients. Ann Epidemiol 
2007;17:584-90.

19. Klabunde CN, Potosky AL, Legler JM, Warren JL. Development of a comorbidity index using physician claims data. J Clin Epidemiol 2000;53:1258-67.
20. Yoon C, Goh E, Park SM, Cho B. Effects of smoking cessation and weight gain on cardiovascular disease risk factors in Asian male population. Atherosclerosis 2010;208:275-9. 
Appendix 1. Association between smoking cessation, weight change, and CVD mortality as well as all-cause mortality

\begin{tabular}{ccccc}
\hline Variable & Total $(\mathrm{N}=36,129)$ & Sustained smoker & \multicolumn{2}{c}{ Ex-smoker } \\
\cline { 4 - 5 } & & & Weight gain $(>5 \mathrm{~kg})$ & No weight gain $(\leq 5 \mathrm{~kg})$ \\
\hline No. of all cause death & 1,036 & 761 & 20 & 255 \\
Unadjusted HR & & Reference & $1.02(0.65-1.58)$ & $1.17(1.01-1.35)$ \\
Adjusted for HR & & Reference & $1.21(0.77-1.90)$ & $0.85(0.73-0.99)$ \\
No. of CVD death & 118 & 88 & 4 & 26 \\
Unadjusted HR & & Reference & $1.76(0.65-4.79)$ & $1.03(0.67-1.60)$ \\
Adjusted HR & & Reference & $2.03(0.73-5.66)$ & $0.73(0.46-1.15)$ \\
No. of CHD death & 47 & 38 & 1 & 8 \\
Unadjusted HR & & Reference & $1.02(0.14-7.41)$ & $0.73(0.34-1.57)$ \\
Adjusted HR & & Reference & $0.96(0.13-7.10)$ & $0.53(0.24-1.17)$ \\
No. of stroke death & 71 & 50 & 3 & 18 \\
Unadjusted HR & & Reference & $2.32(0.72-7.44)$ & $1.26(0.73-2.15)$ \\
Adjusted HR & & Reference & $3.55(1.07-11.70)$ & $0.90(0.51-1.58)$ \\
\hline
\end{tabular}

Values are presented as number or HR (95\% confidence interval).

CVD, cardiovascular disease; HR, hazard ratio; CHD, coronary heart disease.

*Adjusted for CVD risk factors: age, sex, body mass index, systolic blood pressure, total cholesterol, fasting blood glucose, drinking habits, past medical history of hypertension and diabetes, and Charlson comorbidity index. ${ }^{\dagger} \mathrm{CVD}$ comprises $\mathrm{CHD}$ and stroke.

Appendix 2. Association between weight change following smoking cessation and CVD events

\begin{tabular}{|c|c|c|c|c|}
\hline \multirow{2}{*}{ Variable } & \multirow{2}{*}{ Total $(\mathrm{N}=36,129)$} & \multirow{2}{*}{ Sustained smoker } & \multicolumn{2}{|c|}{ Ex-smoker } \\
\hline & & & Weight gain (>5 kg) & Weight gain $(\leq 5 \mathrm{~kg})$ \\
\hline No. of CVD* events & 1,296 & 980 & 18 & 298 \\
\hline Unadjusted HR & & Reference & $0.71(0.45-1.13)$ & $1.06(0.93-1.21)$ \\
\hline Adjusted $\mathrm{HR}^{\dagger}$ & & Reference & $0.69(0.43-1.11)$ & $0.79(0.69-0.90)$ \\
\hline No. of CHD events & 810 & 609 & 10 & 191 \\
\hline Unadjusted HR & & Reference & $0.63(0.34-1.19)$ & $1.10(0.93-1.30)$ \\
\hline Adjusted HR & & Reference & $0.59(0.32-1.11)$ & $0.82(0.70-0.98)$ \\
\hline No. of stroke $e^{\ddagger}$ events & 530 & 405 & 8 & 117 \\
\hline Unadjusted HR & & Reference & $0.76(0.38-1.54)$ & $1.01(0.82-1.24)$ \\
\hline Adjusted HR & & Reference & $0.80(0.39-1.62)$ & $0.73(0.59-0.91)$ \\
\hline
\end{tabular}

Values are presented as number or HR (95\% confidence interval).

CVD, cardiovascular disease; HR, hazard ratio; CHD, coronary heart disease.

${ }^{*} \mathrm{CVD}$ comprises CHD and stroke. ${ }^{\dagger}$ Adjusted for CVD risk factors: age, sex, body mass index, systolic blood pressure, total cholesterol, fasting blood glucose, drinking habits, past medical history of hypertension and diabetes, and Charlson comorbidity index. ${ }^{\ddagger}$ Stroke comprises ischemic stroke and hemorrhagic stroke. 